\title{
Train Monitoring using GSM-R Based Passive Radar
}

\author{
Kevin Chetty ${ }^{\dagger}$, Qingchao Chen ${ }^{\dagger}$ and Karl Woodbridge* \\ ${ }^{\dagger}$ Department of Security and Crime Science \\ *Department of Electronic and Electrical Engineering \\ University College London \\ London, United Kingdom \\ k.chetty@ucl.ac.uk
}

\begin{abstract}
Train detection technologies are universal to all modern railway signal and control systems. They are essential for managing the movement of vehicles across entire transport networks, and to ensure their safe operation. In this paper we investigate the feasibility of a new train monitoring capability based on passive radar technology. The system exploits signal transmissions from the railways' GSM-Railway (GSM-R) radio communications infrastructure, and has the potential to determine the positions and velocities of trains over any section of a railway network where there is GSM-R coverage. A theoretical ambiguity function analysis on directly measured GSM-R waveforms suggest that targets can be detected with axial range resolutions of approximately $850 \mathrm{~m}$, and velocities down to less than $1 \mathrm{mph}$. To demonstrate proof-of-concept, a series of experiments were carried out using a software-defined GSM-R passive radar system. The results show the first detections of trains at bistatic ranges of just over $1 \mathrm{~km}$ moving at various speeds. There are now hundreds of thousands of miles of railway track covered by GSM-R globally, with many more countries planning to rollout systems nationally. The results therefore imply that GSM-R based passive radar technology could be used to develop low-cost train monitoring capabilities worldwide alongside the existing GSM-R radio communications infrastructure.
\end{abstract}

\section{INTRODUCTION}

Train detection technologies are vital for the management of railways, and to ensure their safe operation. Information from such systems can be used to assist in directing railway traffic; in warning systems which alert trackside workers of approaching trains; and to regulate speeds over different sections of track.

Simple train detection systems based around track circuits and treadles are still is use today because of their low-cost, ease of deployment, and simplicity of operation. They can also be used in condition monitoring, for example to detect broken rails. However track circuits can suffer from a number of issues including low-precision detection, right- and wrongside failures caused by contaminants on railheads, rusting, leaves on the track, and wideband interference currents arising from traction [1]. Newer axle-counters are less vulnerable to electromagnetic interference and also remove the need for insulated block joints which are a major source of failure for track circuits. However axle counters are more expensive, require long installation times, and are best suited to lower density routes.

In recent years a number of low-cost technological solutions have been proposed and developed to address the problem of accurately measuring the positions and speeds of trains on the railways. These include fixing accelerometers onto the track [2], coding and transmitting signals measured in track circuits [3], and systems based on RFID technology [4, 5], and wireless sensor networks (WSNs). WSNs have gained popularity for train detection and condition monitoring applications [6,7] due to their use of small and low-cost sensors which can scale-up to monitor large sections of railway networks, and with minimal disturbance to the environments in which they are deployed. WSNs based on inertial sensors to monitor moving trains have also attracted significant research interest, and in [8] the authors present results from a WSN consisting of 3D MEMS accelerometers attached to the railway tracks for capturing the vibrations generated by passing trains.

Global System for Mobile Communications - Railway (GSM-R) has now been adopted across five continents as the standard communication protocol for railway-specific voice and data operational services. In this paper we investigate a new train detection system based on passive radar technology which utilises the railways' GSM-R radio communication network as its illumination source. Passive bistatic radar (PBR) for non-cooperative detection and tracking applications has experienced a resurgence of interest in recent years due to the rapidly growing number available of transmitters of opportunity; increased accessibility to low-cost computational power; and off-the-shelf solutions for synchronizing receiver channels. PBR research examining the use of newer communications protocols that employ digital modulation schemes has been especially noteworthy and include GSM transmissions [9] DAB and DVB-t [10] WiMAX [11] and WiFi [12, 13]. The receive-only nature of passive radar systems means they are low-cost, and not subject to spectrum license fees and are covert in nature which makes them attractive for large scale surveillance and monitoring. Moreover their motion sensitivity is typically orders of magnitude more accurate compared to conventional active 
microwave radars [14] owing to the increased integration times possible, which can aid Doppler tracking techniques.

In this paper we examine the feasibility of GSM-R based passive radar for detecting trains on any section of a railway network where there is GSM-R coverage. Section II outlines the GSM-R specifications relevant to PBR and describes the results from an ambiguity function analysis that was applied to measured GSM-R waveforms in order to assess their detection capability. In Section III we explain the key features of our software-defined GSM-R passive radar prototype system and associated signal processing. Section IV then describes a set of experimental field trials designed to show the feasibility of the technique, before presenting the results in Section V. Finally, Section VI discusses the results and their implications for a new train detection system, and outlines our future work plan.

\section{GSM-R CHARACTERISTICS FOR PASSIVE RADAR}

\section{A. Technical Specifcations and Railway Deploymemt}

GSM-R is built around the GSM-900 specification and provides additional channels and specialized services for use by railway personnel. It enables higher train speeds and traffic density with an increased level of safety. GSM-R is a constituent part of ERTMS (European Rail Traffic Management System) and based on cellular GSM and EIRENE specifications. It is also a Time Division Multiple Access (TDMA) system that employs Gaussian Minimum Shift Keying (GMSK) modulation for data transmission, and operates in the lower extension of the $900 \mathrm{MHz}$ frequency: $876-915 \mathrm{MHz}$ in the uplink, and $921-960 \mathrm{MHz}$ in the downlink. Channel spacing of GSM-R is $200 \mathrm{kHz}$. By 2016, GSM-R is expected to have been selected by 56 countries across 5 continents as their National railway communications standard. It is implemented using base transceiver stations (BTS) that are placed next to railway lines with intervals of approximately 8-20 kilometers. BTS transmission powers are usually around $30 \mathrm{~W}$ and are located on communications towers 20 to 45 meters high, typically with $18 \mathrm{dBi}$ directional antennas.

\section{B. Ambiguity Function Analysis}

The self-ambiguity function (Self-AF) shown in (1) represents the response of a matched filter to the signal for which it is matched, as well as to mismatched Doppler shifted signals [15], and provides an indication of the radar detection characteristics of various waveforms.

$$
\chi_{\text {self }}(\tau, f)=\left|\int_{-\infty}^{\infty} s(t) s *(t-\tau) \exp (j 2 \pi f t) d t\right|^{2}
$$

where $s(t)$ is the signal in the time $t$ domain, $\tau$ is a target range delay and $f$ is the target Doppler shift. To determine the ability of GSM-R waveforms to detect train targets in range and Doppler, transmission signals from an operational BTS were measured using our GSM-R passive receiver system (see Section III). Each measurement consisted of a 6 second recording sampled at $4 \mathrm{MHz}$, and processed using (1). Fig. 1 illustrates the Self-AF for one of the measured signals. The zero-Doppler cut of the Self-AF plot is shown in Fig. 2 and

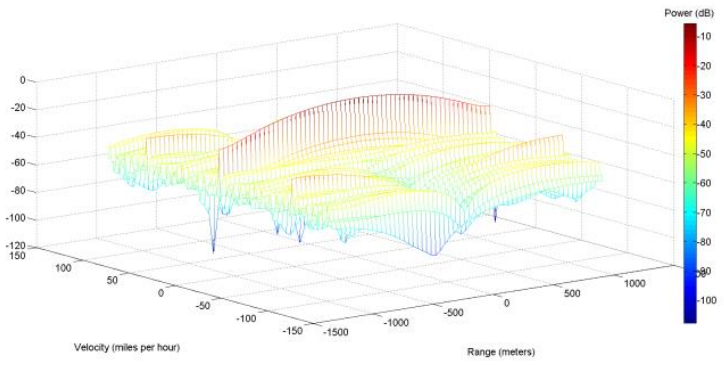

Figure 1. Ambiguity function of a GSM-R downlink signal. The waveform employs a GMSK modulation scheme.

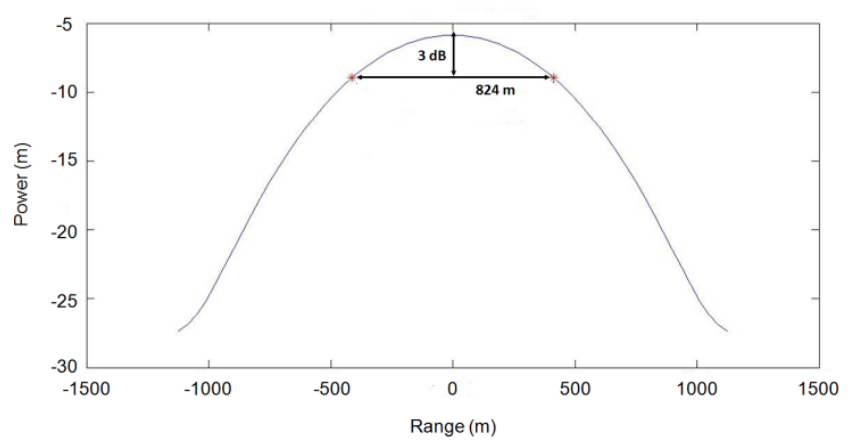

Figure 2. Zero-Doppler cut of ambiguity function shown in Figure 1. The $-3 \mathrm{~dB}$ point on the mainlobe suggests a bistatic range resultion of $824 \mathrm{~m}$.

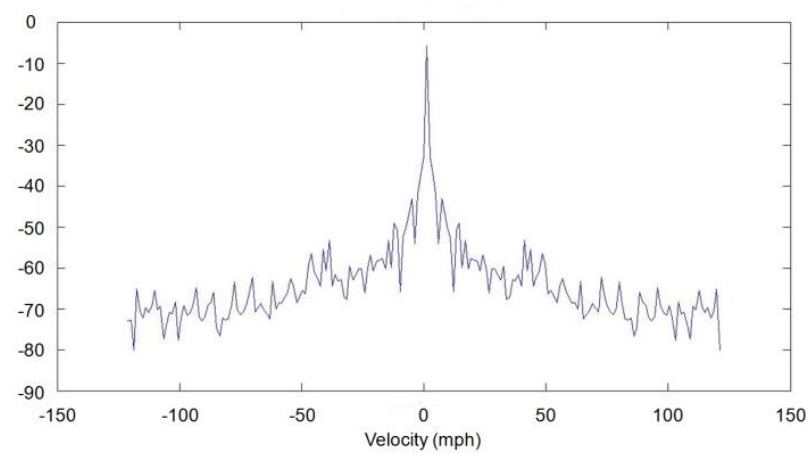

Figure 3. Zero-Range cut of the ambiguity function shown in Figure 1. The $-3 \mathrm{~dB}$ point on the mainlobe suggests a Doppler resultion of $0.167 \mathrm{~Hz}$.

indicates a range-resolution of $825 \mathrm{~m}$. The $6 \mathrm{~s}$ integration time gives a high-Doppler resolution of $0,167 \mathrm{~Hz}$ which equates to a velocity accuracy of better than $1 \mathrm{mph}$ (Fig.3).

\section{SOFTWARE DEFINED GSM-R PASSIVE RADAR}

\section{A. The Universal Software Radio Peripheral}

To demonstrate proof-of-concept, we have developed a GSM-R passive radar based on the Universal Software Radio Peripheral (USRP) hardware platform. The system consists of two USRP-N210's which are each built around a Xilinx ${ }^{\circledR}$ Spartan ${ }^{\circledR}$ FPGA (3A-DSP 3400) and a 100 MS/s, 14-bit ADC. 
In receive, the FPGA performs the digital down conversion and low pass filtering, allowing the recorded data samples to be transferred via the USRP's gigabit Ethernet port to the host laptop. Each USRP motherboard is also fitted with an RFX900 daughterboard which can access the spectral band between 750-1050 MHz. Synchronisation between the boards is achieved using the MIMO expansion port which allows the reference receiver to slave the surveillance receiver by distributing a reference clock and time synchronisation pulse. NI LabVIEWTM software is used to control the system parameters and save the recorded data for offline processing in Matlab. Fig. 4 shows a block diagram of the system.

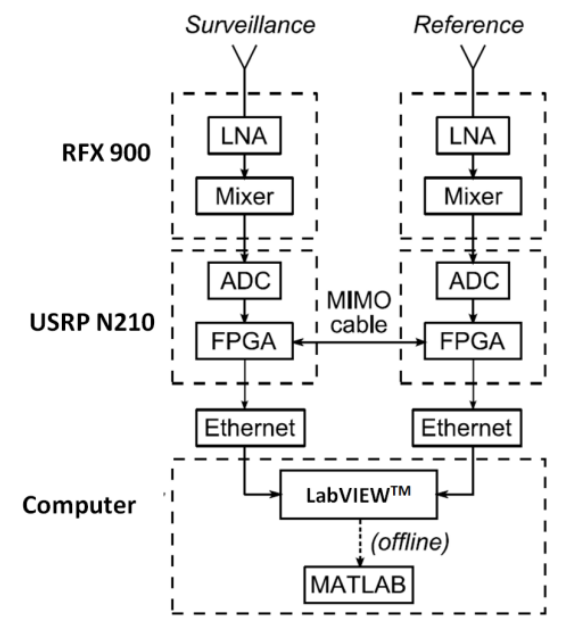

Figure 4. Block diagram of the software-defined GSM-R passive radar.

Once the measured data sets are exported to Matlab, they undergo cross-ambiguity processing described in (2)

$$
\chi_{\text {cross }}(\tau, f)=\left|\int_{-\infty}^{\infty} s(t) u *(t-\tau) \exp (j 2 \pi f t) d t\right|^{2}
$$

where $u(t)$ is the echo signal in the surveillance receiver. Doppler shifts are then translated to target velocities using the bistatic Doppler equation shown in (3)

$$
f_{B}=\frac{2 v \cdot \cos (\beta / 2) \cos \alpha}{\lambda}
$$

where $f_{B}$ is the bistatic Doppler shift, $v$ is the target velocity, $\lambda$ is the wavelength equal to $0.325 \mathrm{~m}, \beta$ is the bistatic angle, and $\alpha$ is the angle between the bistatic bisector and the direction of target motion. Velocity-range surface plots can then be generated from which target responses can be identified.

\section{PROOF-OF-CONCEPT EXPERIMENTS}

\section{A. Experimental Setup}

A series of measurements were taken from a railway footbridge in the vicinity of an operational GSM-R BTS. A 14
$\mathrm{dBi}$ gain, $6.5^{\circ}$ horizontal and $10^{\circ}$ vertical beamwidth reference antenna pointing along one direction of the railway track was used to monitor signal transmissions from the BTS approximately $325 \mathrm{~m}$ away. An identical antenna, also located on the footbridge, was pointed in the opposite direction and used to monitor a surveillance cell approximately $100 \mathrm{~m}$ in length, corresponding to the antenna footprint. Due to the height difference between the GSM-R BTS (which was located on a hill) and the position of the train on the track, the detection geometry formed an approximate bistatic triangle of baseline $325 \mathrm{~m}$, range $1125 \mathrm{~m}$ and angle $18^{\circ}$. A diagram illustrating the experimental setup is shown in Fig. 5. Measurements were taken for various types of train moving at different speeds along both directions of the railway track. However, ground-truth data of the actual velocities are not available for comparison.

\section{RESULTS}

Fourteen 6-second train measurements were taken. Each dataset then underwent cross-ambiguity processing (2) in 0.3 millisecond portions resulting in a series of 20 velocity-range plots (for each measurement). This allowed us to identify the responses of the trains as they passed through and exited the detection cell covered by the surveillance antenna. The results from all fourteen measurements demonstrated the ability of GSM-R passive radar to reliably detect different types of trains, their travel direction, and speed. Fig. 6 shows the results for a fast moving train travelling away from the surveillance receiver at approximately 100 miles per hour. The train is clearly detected at the expected Doppler shift while the zero-Doppler from stationary clutter and direct signal interference (DSI) can also be clearly seen. Fig. 7 shows a similar plot for a slow moving train travelling towards the surveillance receiver. The train can clearly be detected with opposite Doppler shift to Fig 6 despite the much lower velocity. An analysis of the target detection peaks in the measured data indicated a range resolution of approximately $825 \pm 50 \mathrm{~m}$ which is in agreement with the output from the ambiguity function analysis in Section IIB.

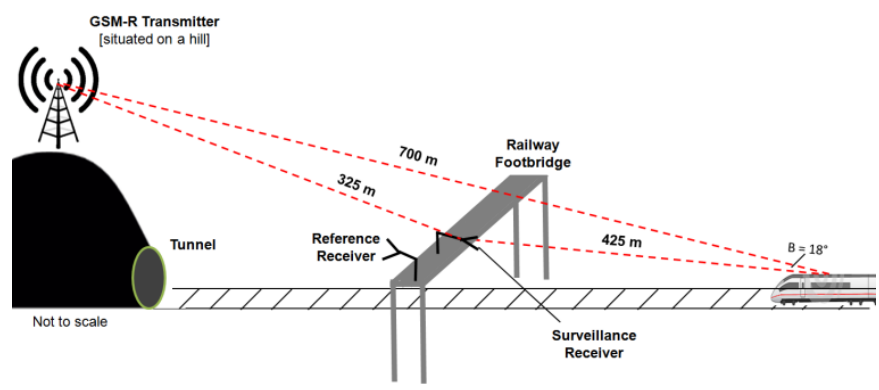

Figure 5. Bistatic geomtery of the train detection experiments. The GSM-R base tranceiver station was located on a hill above a tunnel and illuminated trains approximately $700 \mathrm{~m}$ away. Both the surveillance and reference antennas were located on a railway footbridge. 


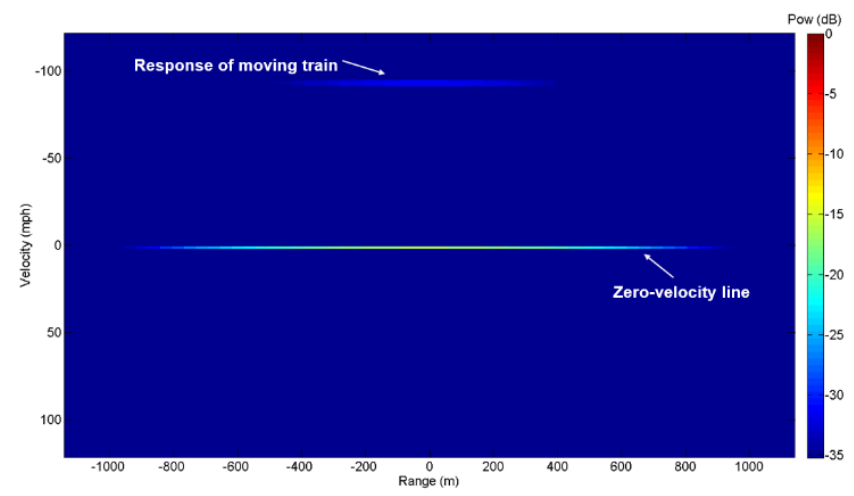

Figure 6. Detection of a fast moving railway train using GSMR-R passive radar. The train is travelling away from the surveillance receiver so has a positive velocty. Note the $\mathrm{x}$-axis has been re-scaled so that the coverage cell defined by the footprint of the surveillance receiver at $1125 \mathrm{~m}$ bistatic range has been set to $0 \mathrm{~m}$ range.

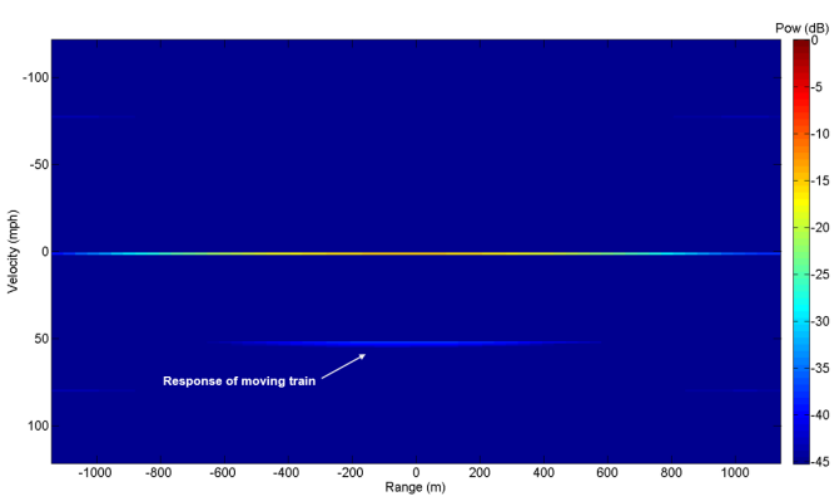

Figure 7. Detection of a slow moving railway train using GSMR-R passive radar

\section{SUMMARY AND DISCUSSION}

The research reported in this paper has investigated the feasibility of detecting trains using passive radar technology exploiting the railways' GSM-R radio communications infrastructure. An ambiguity function analysis was carried out on measured GSM-R waveforms and indicated that a high Doppler resolution is obtainable while range resolution is limited to around $850 \mathrm{~m}$ due to the signal bandwidth. The relatively low sampling overhead associated with the $200 \mathrm{KHz}$ bandwidth also permits long integration times to give even higher Doppler sensitivity.

A series of proof-of-principle experiments were carried on a working railway line using our passive GSM-R radar system built around modular software defined hardware. The results show the first reported detections of trains using GSM-R based passive radar. In all fourteen measurements taken the response of the train could be clearly identified and this high detection reliability is attributed to the high RCS of train targets. However, the DSI gave rise to a strong peak zeroDoppler in the velocity-range surface which increased the dynamic range of the cross-ambiguity processing output and acted to subdue the target response. Future work will therefore focus on applying DSI suppression signal processing techniques $[16,17]$ to the data. We also plan to investigate the ability of GSM-R to monitor personnel movements around the perimeter of a railway track, such as those from railway infrastructure workers, and intruders.

\section{REFERENCES}

J. Palmer, "The need for train detection," in Railway Signalling and Control Systems, 2006. The 11th IET Professional Development Course on, 2006, pp. 47-53.

L. Angrisani, D. Grillo, R. S. L. Moriello, and G. Filo, "Automatic detection of train arrival through an accelerometer," in Instrumentation and Measurement Technology Conference (I2MTC), 2010 IEEE, 2010, pp. 898-902.

[3] K. Oguma, A. Kawabata, K. Tashiro, M. Fujiwara, and S. Tanifuji, "Train detection system and a train detection method," 2006.

[4] B.-K. Cho, "RFID Antenna for Position Detection of Train," in Future Information Technology. vol. 309, J. J. Park, Y. Pan, C.-S. Kim, and Y. Yang, Eds., ed: Springer Berlin, 2014, pp. 903-908.

[5] A. K. Gupta, S. Katiyar, and N. Kumar, "Railway Track Finding System with RFID Application," International Journal of Computer Applications, vol. 83, pp. 24-30, 2013.

S. Gupte, O. Masoud, R. F. K. Martin, and N. P. Papanikolopoulos, "Detection and classification of vehicles," Intelligent Transportation Systems, IEEE Transactions on, vol. 3, pp. 37-47, 2002.

V. Hodge, M. Weeks, S. O'Keefe, and A. Moulds, "Wireless Sensor Networks for Condition Monitoring in the Railway Industry: A Survey," Intelligent Transportation Systems, IEEE Transactions on, vol. 16, pp. 1088 - 1106, 2015.

E. Berlin and K. Van Laerhoven, "Sensor Networks for Railway Monitoring: Detecting Trains from their Distributed Vibration Footprints," in Distributed Computing in Sensor Systems (DCOSS), 2013 IEEE International Conference , 2013, pp. 80-87. R. Zemmari, M. Daun, M. Feldmann, and U. Nickel, "Maritime surveillance with GSM passive radar: Detection and tracking of small agile targets," in Radar Symposium (IRS), 2013 14th International, 2013, pp. 245-251.

[10] K. E. Olsen and K. Woodbridge, "Performance of a multiband passive bistatic radar processing scheme ; Part I," Aerospace and Electronic Systems Magazine, IEEE, vol. 27, pp. 16-25, 2012. K. Chetty, K. Woodbridge, H. Guo, and G. E. Smith, "Passive bistatic WiMAX radar for marine surveillance," in Radar Conference, 2010 IEEE, 2010, pp. 188-193.

[12] K. Chetty, G. Smith, G. Hui, and K. Woodbridge, "Target detection in high clutter using passive bistatic WiFi radar," in Radar Conference, 2009 IEEE, 2009, pp. 1-5.

[13] P. Falcone, F. Colone, A. Macera, and P. Lombardo, "Localization and tracking of moving targets with WiFi-based passive radar," in Radar Conference (RADAR), 2012 IEEE, 2012, pp. 0705-0709.

[14] P. Howland, "Editorial: Passive radar systems," Radar, Sonar and Navigation, IEE Proceedings -, vol. 152, pp. 105-106, 2005.

[15] C. J. Baker, H. D. Griffiths, and I. Papoutsis, "Passive coherent location radar systems," Radar, Sonar and Navigation, IEE Proceedings -, vol. 152, pp. 160-168, 2005.

[16] K. Chetty, G. E. Smith, and K. Woodbridge, "Through-the-Wall Sensing of Personnel Using Passive Bistatic WiFi Radar at Standoff Distances," Geoscience and Remote Sensing, IEEE Transactions on, vol. 50, pp. 1218-1226, 2012.

[17] F. Colone, D. W. O'Hagan, P. Lombardo, and C. J. Baker, "A Multistage Processing Algorithm for Disturbance Removal and Target Detection in PBR," Aerospace and Electronic Systems, IEEE Transactions on, vol. 45, pp. 698-722, 2009. 